\title{
Lupus eritematoso sistémico con mesenteritis retráctil y síndrome antifosfolípido catastrófico
}

\author{
A. MERA VARELA, J. BLANCO RODRÍGUEZ, S. INSUA VILARIÑO,
} M. CAMAÑO FREIRE

Servicio de Reumatología. Complejo Hospitalario Universitario de Santiago. Facultad de Medicina. Universidad de Santiago. Santiago de Compostela

SYSTEMIC LUPUS ERYTHEMATOSUS WITH RETRACTIL MESENTE RITIS AND CATASTHROPHIC ANTIPHOSPHOLIPID SYNDROME

\section{RESUMEN}

El Síndrome Antifosfolípido coexistiendo con Lupus se ha asociado a una amplia variedad de manifestaciones clínicas, la mayor parte de éstas, ligadas a la isquemia o el infarto focal. El fracaso agudo multiorgánico que ocurre en los pacientes con estos anticuerpos se ha llamado Síndrome Antifosfolípido Catastrófico por la alta mortalidad. Por otra parte, el término Mesenteritis Retráctil se aplica a aquella circunstancia en la que un proceso inflamatorio crónico con engrosamiento e infiltración del mesenterio abdominal ocurre, debido a un mecanismo isquémico entre varias múltiples causas. Presentamos un paciente en el que existió la concomitancia de Síndrome Antifosfolípido, isquemia de órganos abdominales y Mesenteritis Retráctil. El paciente desarrolló un síndrome antifosfolípido catastrófico, probablemente secundario a un proceso infeccioso, y tras la supresión de la terapia antitrombótica debido a la presencia de una hemorragia digestiva alta, falleciendo.

PALABRAS CLAVE: Mesenteritis retráctil. Paniculitis abdominal. Síndrome Antifosfolípido Catastrófico. Lupus Eritematoso Sistémico.

\begin{abstract}
The Antiphospholipid Syndrome coexisting with Lupus has been associated to wide variety of clinical manifestations, mostly linked to focal ischaemia or infarction. Acute multi-organ failure occurring in patients with these autoantibodies is named Catastrophic Antiphospholi pid Syndrome because the high mortality. On the other hand, the term Retractile Mesenteritis is applied to the circumstance in which, an chro nic inflammatory process with thickening and infiltration of the mesente rium occur, due to ischaemic mechanism, among many other multiple causes. We describe one patient with concomitance of Antiphospholipid Syndrome, evidence of abdominal ischaemia and Retractile Mesenteritis. The patient developed Catastrophic Antiphospholipid Syndrome, pro bably due to infectious process and, antithrombotic therapy supression because upper gastrointestinal bleeding, and dead.
\end{abstract}

KEY WORDS: Retractile Mesenteritis. Panniculitis. Catastrophic Antiphospholipid Syndrome. Systemic Lupus Erythematosus.

Mera Varela A, Blanco Rodríguez J, Insua Vilariño S, Camaño Freire M. Lupus eritematoso sistémico con mesenteritis retráctil y síndrome antifosfolípido catastrófico. An Med Interna (Madrid) 2001; 18: 143-146.

\section{INTRODUCCIÓN}

El Síndrome Antifosfolípido (SAF), se ha asociado con la presentación de trombosis arterial o venosa, pérdidas fetales recurrentes, trombopenia, y con menor frecuencia, a otras complicaciones (1). Algunos pacientes con anticuerpos antifosfolípido pueden desarrollar un cuadro, a menudo de desenlace fatal, denominado Síndrome Antifosfolípido Catastrófico (SAFC), que cursa con fallo multiorgánico secundario a trombosis masiva y en el que predomina la afectación renal, pulmonar, gastrointestinal, suprarrenal y del sistema nervioso central (2), existiendo varias descripciones del mismo en la literatura médica de los últimos años. El desen- cadenante final de los eventos trombóticos generalizados que caracterizan a este síndrome es desconocido, si bien, entre sus posibles causas se han comunicado la presencia de infección concomitante, el abandono de la terapia anticoagulante o el estado postoperatorio de determinadas intervenciones quirúrgicas (3).

Por otra parte, la mesenteritis retráctil (MR) o paniculitis abdominal es una rara entidad, de etiología incierta, caracterizada por una inflamación inespecífica del tejido adiposo mesentérico, que evoluciona hacia la fibrosis y retracción (4).

Presentamos un caso de Lupus Eritematoso Sistémico (LES) que desarrolló ambas manifestaciones, SAF y MR, con un curso crónico y un desenlace fatal, en forma de SAFC.

Trabajo aceptado: 15 de Julio de 1999

Correspondencia: A. Mera Varela. Servicio de Reumatología. Complejo Hosp. Universitario de Santiago. C/ Galeras s/n. 15705 Santiago de Compostela. A Coruña 


\section{CASO APORTADO}

Varón de 38 años, albañil de profesión, con antecedentes de fumador de 5 cigarrillos/día, que en 1990 consulta por desarrollar un cuadro de astenia, dolor pleurítico y artralgias, de algunos días de evolución, acompañados de pancitopenia en una analítica rutinaria. En la radiología de tórax se apreciaba pequeño derrame pleural y las determinaciones de ANA sobre Hep-2 y Anti-DNA resultaron positivas, por lo que fue diagnosticado de LES, iniciandose tratamiento con esteroides y azatioprina, con buena respuesta, permaneciendo asintomático durante 2 años.

En 1992, mientras estaba recibiendo tratamiento con 30 mgrs. de Prednisona en días alternos, comienza, de forma brusca, a desarrollar alteraciones conductuales, seguidas por alucinaciones visuales y delirio, precisando ingreso hospitalario. En este momento, la exploración mostró febrícula, valores normales de tensión arterial y ausencia de focalidad neurológica o déficits visuales, con normalidad de las determinaciones habituales del LCR. Se practicó Resonancia Magnética, que, como único hallazgo, mostraba una pequeña área de hiperseñal en la sustancia blanca de la región frontoparietal. Entre los datos analíticos destacaban, la presencia de leucopenia, anemia y trombopenia $\left(62 \times 10^{3}\right.$ $\mathrm{mm}^{3}$; normal: $135-369 \times 10^{3} \mathrm{~mm}^{3}$ ), $\mathrm{C}^{\prime} 3 \mathrm{c}$ : $74 \mathrm{U} / \mathrm{ml}$. (normal: $90-180$ $\mathrm{mg} / \mathrm{dl}), \mathrm{C} 4: 14 \mathrm{U} / \mathrm{ml}$.(normal: 10-40 mg/dl.), Anti-DNA $110 \mathrm{U} / \mathrm{ml}$ (n: $<25 \mathrm{U} / \mathrm{ml}$ ) y ENA (Antígenos Nucleares Extraíbles) y anticuerpos anti-ribosoma $\mathrm{P}$ negativos. Los anticuerpos anticardiolipina (aCL-IgG) fueron informados como positivos, sin cuantificación. En los días siguientes presentó dolor abdominal difuso y distensión, realizándose un examen ecográfico que reveló litiasis biliar sin datos de obstrucción, disminución en el tamaño hepático, con heteroecogenicidad y borde festoneado. No se objetivaron datos de trombosis, pero el sistema portal se encontraba algo dilatado, con esplenomegalia, ascitis y ligero derrame pleural derecho. El examen del líquido ascítico mostraba 500 células/mm3 (70\% PMN); Proteinas 4,5 gr/l; Glucosa $88 \mathrm{mgr} / \mathrm{dl}$; LDH $450 \mathrm{U} / \mathrm{l}$; los cultivos y la tinción de Ziehl-Nielsen del líquido ascítico fueron negativos. El Mantoux fue también negativo. Se practicó biopsia hepática por laparoscopia, que reveló un tejido macroscópicamente normal. En el examen microscópico aparecían áreas de colagenización y depósitos de lipofucsina en hepatocitos cercanos a áreas perivenulares, se apreciaba ligera dilatación de los sinusoides y las tinciones para amiloide resultaron negativas. El paciente fue tratado con esteroides ( $80 \mathrm{mgr} / \mathrm{día})$, plasmaféresis, ciclofosfamida, aspirina y neurolépticos, con buena respuesta, experimentando una rápida desaparición de las manifestaciones psiquiátricas en 10 días y mejoría progresiva del dolor abdominal, la ascitis y la distensión.

Un año después, presentó un nuevo episodio de dolor abdominal no febril, con un área dolorosa a la palpación en el hemiabdomen derecho y desarrollo de una induración objetivable con la palpación profunda. El estudio ecográfico reveló ligera ascitis y presencia de septos en el peritoneo (Fig. 1) En el enema opaco y en el tránsito intestinal baritado, se apreció un engrosamiento de las válvulas conniventes y una disminución en el diámetro del colon y del recto-sigma, sin hallazgos radiológicos sugestivos de enfermedad inflamatoria intestinal. La tomografía axial (TAC) abdominal mostraba ascitis loculada, disminución del tamaño hepático con áreas sugestivas de isquemia focal, esplenomegalia y litiasis biliar. El sistema portal se encontraba permeable, pero con una ligera irregularidad en su pared que sugería trombosis previa recanalizada, destacando la presencia de aumento en la densidad y engrosamiento del mesenterio. En el estudio arteriográfico se evidenciaba una irregularidad en el sistema arteriolar hepático, con incompleta visualización del lecho portal y derivación del flujo hacia el sistema iliaco-cava izquierdo. La biopsia rectal fue negativa para vasculitis y amiloide. Una nueva determinación de aCL-IgG fue de 25 GPL (> 2 desviaciones estándar). Se inició terapia antitrombótica, y de acuerdo con el paciente se creyó que en ese momento no era susceptible de anticoagulación com- pleta por el tipo de trabajo que desarrollaba, y en espera de valoración de la respuesta a la misma. La trombopenia se resolvió y el paciente permaneció prácticamente asintomático durante los años siguientes, con algunos episodios de dolor abdominal por crisis suboclusivas autolimitadas, que se resolvían en unas 24 horas con la instauración de dieta absoluta, y mantenía dosis bajas de esteroides y aspirina como terapia.

Ingresa por última vez en nuestro Servicio, por episodio febril, con diarrea y dolor abdominal difuso, sin datos exploratorios de peritonismo. Entre los datos analíticos al ingreso destacaban: 4,43×10 Hematíes (normal: 4,5-5,5x10 $\mathrm{mm}^{3}$ ), $\mathrm{Hb} 12,8 \mathrm{~g} / \mathrm{dl}($ normal:13,5-17,5 $\mathrm{mg} / \mathrm{dl}$ ), Leucocitos $5,620 \times 10^{3} / \mathrm{mm}^{3}$ (normal: $4,09-10,08 \times 10^{3} / \mathrm{mm}^{3}$ ), Plaquetas $44 \times 10^{3} \mathrm{~mm}^{3}$ (normal:135-369x $10^{3} \mathrm{~mm}^{3}$ ) Urea $54 \mathrm{mg} / \mathrm{dl}$ (normal: 12-44), Creatinina 0,7 mg/dl (normal: 0,4-1,3), Amilasa 85 $\mathrm{mg} / \mathrm{dl}$ (N: 50-250), CK 29 U/l (normal: 15-110), Na $142 \mathrm{mEq} / \mathrm{l}$ (normal: $137-148$ ), K $3,7 \mathrm{mEq} / 1$ (normal: $3,6-4,9)$, Ca $8,3 \mathrm{mg} / \mathrm{dl}$ (normal: 8,7-10,6), Protrombina 91\% (normal: 75-100\%), TTPA 25,3 segundos (N 23-37). Se programó tratamiento con antibioterapia empírica (Clindamicina y Tobramicina), dieta absoluta, metilprednisolona a dosis de $40 \mathrm{mgr} / \mathrm{día}$ y se mantuvo la aspirina a dosis antiagregantes, con mejoría clínica inicial. Durante el tercer día de ingreso, comenzó a experimentar un progresivo deterioro del estado general, con exacerbación del dolor abdominal, hipotensión progresiva y shock. Los hemocultivos fueron positivos para Klebsiella pneumoniae, sensible a los antibióticos que se habían instaurado previamente. Con la hipótesis de shock séptico fue trasladado a la Unidad de Cuidados Intensivos. Durante los 9 días de estancia en esta Unidad, presentó un episodio de hemorragia digestiva alta con ulceración múltiple a nivel gástrico en una endoscopia, y un cuadro de distress respiratorio, siendo suspendida la terapia antiagregante. Tras la supresión, una nueva analítica revelaba: Hematíes 3,6×106, Hb 9,4 g/dl, Leucocitos $32 \times 10^{3} \mathrm{~mm}^{3}$ y Plaquetas $8 \times 10^{3} \mathrm{~mm}^{3}$; Creatinina $0,9 \mathrm{mg} / \mathrm{dl}$; Protrombina $33 \%$ y TTPA 48,7 segundos. El resto de los parámetros eran normales, a excepción de una hipoalbuminemia. El paciente desarrolló entonces una hemiparesia derecha y posteriormente, coma neurológico con TAC que mostró infarto cerebral masivo con edema cerebral secundario, falleciendo. Se realizó estudio necrópsico, que demostró una trombosis extensa en el parénquima cerebral, trombosis intestinal arterial y venosa, con engrosamiento y formación de adherencias y septos en el mesenterio y peritoneo (Fig. 2).

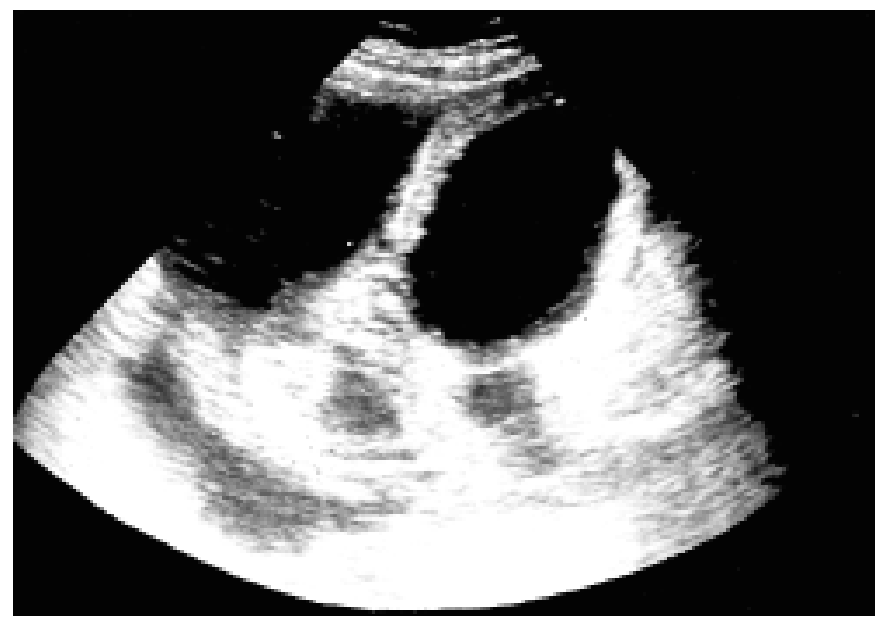

Fig. 1. Ecografía en sección longitudinal que muestra ascitis con ecos en su interior y una imagen quística de pared engrosada y contenido mixto, correspondiendo a ascitis septal. 


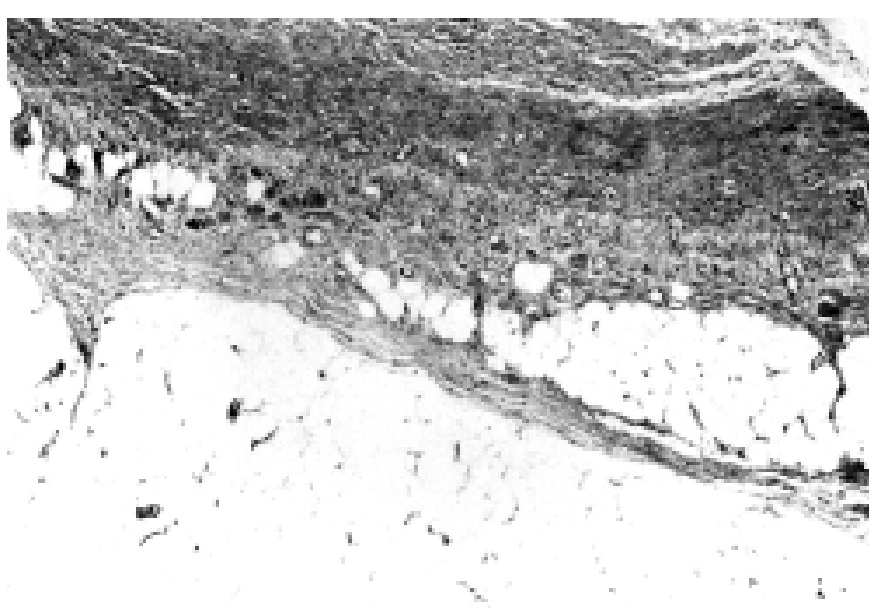

Fig. 2. Imagen microscópica del mesenterio. Paniculitis septal con infiltrado celular y moderada fibrosis mesentérica. (Hematoxilinaeosina; $x 20$ )

\section{DISCUSIÓN}

En el Lupus puede presentarse ascitis de forma aguda o crónica, pero es extremadamente rara la ascitis prominente. La forma aguda ocurre en el $11 \%$ de los casos y la crónica, en general de curso indolente, clínicamente es mucho menos frecuente de detectar, si bien se ha comunicado hasta en $2 / 3$ de los pacientes con LES en alguna ocasión (5). La presencia de ascitis es común en algunas enfermedades del peritoneo y mesenterio en las que la fibrosis y la retracción son las manifestaciones predominantes. Jura, ya en 1924 (6) sugería el término "paniculitis mesentérica" para aquellos casos con inflamación mesentérica aguda o subaguda, y el de "mesenteritis retráctil", también denominada "lipogranulomatosis esclerosante", para el proceso inflamatorio crónico complicado con fibrosis y retracción del peritoneo y el mesenterio. Esta entidad ha sido considerada como el estadío final inespecífico de una amplia variedad de procesos abdominales, sobre todo, del tejido adiposo mesentérico, con desarrollo posterior de fibrosis y retracción (4). Los hallazgos histológicos incluyen: anormalidad de las células adiposas con citoplasma espumoso, e infiltrado de monocitos, linfocitos y macrófagos cargados de lípidos. En algunos casos se aprecian células gigantes de cuerpo extraño, áreas de necrosis grasa y tejido colágeno fibroso que sustituye al tejido graso (7).

La relación con otras patologías, previas o concomitantes, incluye: cirugía abdominal, pancreatitis, cirrosis hepática, colelitiasis, linfosarcoma gástrico y otras neoplasias (7). Han (8) y Rigaud (9) han propuesto un mecanismo de isquemia crónica entre otras etiologías, para explicar este proceso. Hasta el momento actual, en nuestro conocimiento, no se ha descrito ningún caso de Lupus o SAF con este tipo de patología, siendo probablemente éste el primer caso documentado de MR en que, tras exclusión de otras causas, se puede achacar a mecanismo isquémico secundario a SAF y LES.

Las alteraciones características en la TAC y en otros estudios de imagen son de gran ayuda para el diagnóstico, pero el diagnóstico diferencial debe realizarse, sobre todo, con la fibrosis retroperitoneal $(10,11)$. En el enema opaca y el trán- sito intestinal baritado puede observarse desplazamiento de las asas intestinales por una masa extrínseca, dilatación de asas aisladas, fijación, estrechamiento de la luz, prolongación del tiempo de tránsito, y, en algunos casos, obstrucción intestinal. Los resultados de la ecografía varian desde áreas hipoecoicas de forma irregular, a masas hiperecoicas con áreas hipoecoicas en el centro (12). Las alteraciones evidenciadas en la TAC se correlacionan con los hallazgos quirúrgicos o necrópsicos macroscópicos, y pueden corresponder a masas localizadas de densidad grasa, interpuestas con otras de densidad tejido blando sobre el mesenterio, con densos haces neurovasculares, engrosamientos focales de la pared intestinal y calcificaciones ocasionales. La proporción de densidad grasa y densidad tejido blando en la TAC, puede variar de acuerdo con la proporción de grasa y tejido fibroso en el estudio histológico (13).

Para el diagnóstico de mesenteritis intrabdominal, se consideran necesarios los siguientes criterios: a) áreas de tejido graso, difusas, únicas o múltiples, con apariencia de masas distribuidas por el mesenterio, retroperitoneo, omentun y/o pelvis, b) confirmación histológica de necrosis grasa con infiltrado inflamatorio y/o infiltración por macrófagos cargados de lípidos espumosos, y c) ausencia de datos histológicos de pancreatitis, enfermedad inflamatoria intestinal o necrosis grasa extrabdominal (14). En nuestro paciente, los hallazgos radiológicos y ecográficos resultaron compatibles con este raro proceso, siendo confirmados por los resultados de los estudios histológicos de la necropsia.

Por otra parte, el paciente presentaba un SAF documentado por la presencia de positividad para los Anticuerpos Anticardiolipina, obstrucción del flujo vascular abdominal y trombopenia. El hígado y otros órganos abdominales no son excepciones en estas circunstancias, y no sólo los grandes vasos como la arteria o vena hepática pueden ser semiocluidos, sino también pequeños vasos pueden producir una amplia variedad de anomalías clínicas y serológicas (15),. Nuestro paciente presentó unas alteraciones ecográficas y en la TAC sugestivas de infartos, asi como heterogeneidad en el parénquima hepático, con borde festoneado, que sugieren enfermedad oclusiva venosa. La indicación absoluta para una completa anticoagulación fue rechazada por nuestro paciente y considerada, por nuestra parte, como de "alto riesgo", debido a antecedentes de pequeños traumatismos frecuentes, si bien la aspirina fue una buena opción para prevenir eventos trombóticos mayores, pero probablemente no suficiente para evitar un estado "isquémico crónico" y una mesenteritis retráctil secundaria.

Cuando el paciente padeció una infección aguda (descrita como causa de un SAFC en un 40\% de los casos), se precipitó un proceso de hipercoagulación con un sangrado gastrointestinal concurrente, por lo que se suspendió la aspirina, con el desarrollo posterior de eventos trombóticos extensos, especialmente en el sistema nervioso central, que fue la causa final del fallecimiento (16).

Presentamos este caso de LES con isquemia crónica de órganos abdominales que creemos que es la causa del desarrollo de la mesenteritis, con la aparición final de un SAFC, que derivó en la muerte del paciente como consecuencia de la necesidad de suspender la antiagregación en presencia de hemorragia digestiva alta, creando una situación de "callejón sin salida" entre la decisión de mantener la antiagregación y la suspensión de la misma por presencia de hemorragia digestiva. 


\section{Bibliografía}

1. Harris EN, Gharavi A, Asherson RA, Khamashta M, Hughes GRV. Antiphospholipid antibodies:middle aged but robust. J Rheumatol 1994; 21: 978-981.

2. Asherson RA. The catastrophic antiphospholipid syndrome. J Rheumatol 1992;19: 508-512.

3. Asherson RA, Piette JC. The catastrophic antipospholipid syndrome 1996: acute multiorgan failure associated with antiphospholipid antibodies: a review of 31 patients. Lupus 1996; 5: 414-417.

4. Tytgat G N, Roozendal K, Winter W, Esseveld M R. Successful treatment of a patient with retractile mesenteritis with prednisone and azathioprine. Gastroenterology 1980; 79: 352-356.

5. Hammondeh M, Rahim Siam A. Recurrente peritonitis with ascites as the predominant manifestation of systemic lupus erythematosus. Clin Rheumatol 1995; 14: 352-354.

6. Jura V. Sulla mesenterite retrattile e sclerosante. Policlinico (Prot) 1924; 31: 575.

7. Durst AL, Freund H, Rossemman E, Birnbaum D. Mesenteric panniculitis: review of the literature and presentation of cases. Surgery 1977; 81: 203-211.

8. Han SY, Koehler RE, Keller FS, Ho KJ, Zornes SL. Retractile mesenteritis involving the colon: pathologic and radiologic correlation (case report). AJR 1986; 147: 268-270.

9. Rigaud C, Bogomoletz WV. Association mésentérite rétractile et entérite ischémique. Ann Pathol 1983; 3:171-173.

10. Katz ME, Heiken JP, Glazer HS, Lee JKT. Intrabdominal panniculitis Clinical, radiographic and CT features. AJR 1985; 145: 293-296.

11. Kelly JK, Hwang WS. Idiopatic retractile (sclerosing) mesenteritis and its differencial diagnosis. Am J Surg Pathol 1989; 13: 513-521.

12. Pérez-Fontán J, Soler R, Sánchez J, Iglesias P, Sanjurjo P, Ruiz J. Retractile mesenteritis involving the colon: barium enema, sonographic and CT findings AJR 1986; 147: 937-940.

13. Shu-Hang NG, Ho-Fai Wong, Sheung-Fat Ko, Chung-Cheung Tsai. Retractile mesenteritis with colon and retroperitoneum involvement: CT findings. Gastrointest Radiol 1992; 17: 333-335.

14. Hartz R, Stryker S, Sparberg M, Poticha SM. Mesenteric tumefactions. Am Surg 1980; 46: 525-529.

15. Asherson RA, Khamashta MA, Hughes GRV. The hepatic complications of the antiphospholipid antibodies. Clin Exp Rheumatol 1991; 9: 341-344.

16. Levine SR, Brey RL. Neurological aspects of antiphospholipid antibody syndrome. Lupus 1996; 5: 347-353. 